\title{
Isomon instabilities driven by energetic ions in Wendelstein 7-X
}

\author{
Ya.I. Kolesnichenko ${ }^{1}$, A. Könies ${ }^{2}$, V.V. Lutsenko ${ }^{1}$, \\ M. Drevlak ${ }^{2}$, Yu. Turkin² ${ }^{2}$ P. Helander ${ }^{2}$ \\ ${ }^{1}$ Institute for Nuclear Research, Prospekt Nauky 47, Kyiv 03028, Ukraine \\ ${ }^{2}$ Max-Planck-Institut für Plasmaphysik, Wendelsteinstraße 1, D-17491, Greifswald, \\ Germany
}

\begin{abstract}
It is found that modes of Alfvénic character affected by plasma compressibility and having equal poloidal and toroidal mode numbers (named "isomon modes") can exist in W7-X. These modes, and the conditions under which they arise, are sensitive to the magnitude of the rotational transform of the field lines and the presence of energetic ions. The energetic ions produced by neutral-beam injection (having the energy $55-60 \mathrm{keV})$ interact resonantly with large-scale isomon modes $(m=n \ll 10)$, which tends to lead to instabilities extending over a large part of the plasma cross section.
\end{abstract}

Submitted to: Nuclear Fusion 


\section{Introduction}

Several types of plasma instabilities driven by energetic ions, with exhibiting a range of various consequences, have been observed in toroidal fusion facilities. In particular, instabilities excited by the energetic ions produced during plasma heating by Neutral Beam Injection (NBI) were observed in the Wendelstein 7-AS stellarator. In this device, beam-driven instabilities occurred in both low-beta discharges and high-beta discharges; however, in the latter case they were transient and occurred after switching to full NBI power and during density ramp up [1-3]. It is of great interest to predict the influence of energetic ions on plasma stability in Wendelstein 7-X, the next-step optimized stellarator of the same line. This question has motivated us to carry out the present work contributing to the study of plasma stability during NBI in W7-X.

The magnetic configuration of $\mathrm{W} 7-\mathrm{X}$ has a few peculiarities requiring particular attention. Like its predecessor, W7-AS, the field of W7-X is characterized by small magnetic shear, but the rotational transform, $\iota$, is close to unity in contrast to W7-AS, where $\iota$ was in the range $0.3-0.5$, see figure 1 . Note that $\iota$ in a Helias reactor (a Wendelstein-line device) may be close to unity, too [4]. One can expect that this peculiarity of W7-X and the Helias reactor will lead to new features of Alfvén eigenmodes and Alfvén-sound modes as compared with those in W7-AS.

One of these features was revealed in [5] (see also the overview [6]), where it was shown that the local (continuum) frequency of the Geodesic Acoustic Mode, GAM, $\left(\omega_{G A M}\right)$ in W7-X only slightly exceeds the sound frequency $\left(\omega_{s}=\iota c_{s} / R\right.$, where $c_{s}$ is the sound velocity and $R$ is the major radius of the torus) across the entire plasma crosssection because the so-called "sound parameter", $\mathcal{S}$, is not small $\left(\mathcal{S} \propto \iota^{2}\right)$. In contrast, the GAM frequency well exceeds the sound frequency in devices with $q \equiv \iota^{-1} \gg 1$.

Despite the fact that $\omega_{G A M} \sim \omega_{s}$ in W7-X, one cannot rule out that GAMs will be destabilized by energetic ions in $\mathrm{W} 7-\mathrm{X}$, because the damping rate caused by the bulk ions is relatively small, $\gamma_{d a m p} \ll \omega_{G A M}$. The point is that the phase velocity $\left(\omega / k_{\|}\right.$, where $\omega$ is the wave frequency, $k_{\|}$is the wavenumber along the magnetic field) well exceeds the ion thermal velocity, $v_{T i}$, even when the wave frequency only slightly exceeds the sound frequency. Indeed, for $\omega=\kappa_{s} \omega_{s}$ with $\kappa_{s}>1$, we have $\omega^{2} /\left(k_{\|}^{2} v_{T i}^{2}\right)=\kappa_{s}^{2} c_{s}^{2} / v_{T i}^{2}=\kappa_{s}^{2} \Gamma \gg 1$, with $\Gamma$ is the specific heating ratio. Another reason why one can expect that the damping will be relatively small is that $k_{\|}$equals to $\iota / R$ only for one of the components of the GAM mode, whereas $k_{\|}=0$ for another main component. In addition, experiments on the NSTX spherical tokamak showed that the BAAE mode (Beta-induced Alfvén-acoustic Eigenmode) in the sound-frequency range was destabilized by injected fast ions [7].

On the other hand, because $\iota$ is close to unity and the shear is small, new modes may appear in W7-X. For instance, Alfvénic-type modes with a global structure and frequency above the sound frequency but below the frequency of toroidicity-induced Alfvén eigenmodes, $\omega_{T A E}$, may arise. To understand why, let us assume that $m=n \gtrsim 1$ and $\omega=k_{\|} v_{A}$, where $m$ and $n$ denote the poloidal and toroidal mode numbers, respectively, and $v_{A}$ the Alfvén velocity. Then $\omega_{m=n} \sim m \Delta \iota v_{A} / R$ and $\omega_{m=n} / \omega_{s} \sim$ $m \Delta \iota /\left(\iota \sqrt{\beta_{i}}\right)$, where $\Delta \iota=1-\iota, \beta_{i}$ is the ion beta $\left(8 \pi p_{i} / B^{2}\right)$. For $\beta_{i} \sim 0.01$ and $\Delta \iota / \iota=0.1$ we obtain $\omega_{m=n} / \omega_{s} \sim m$. On the other hand, $\omega_{m=n} / \omega_{T A E}=2 m \Delta \iota / \iota<1$ for $m<5$. 


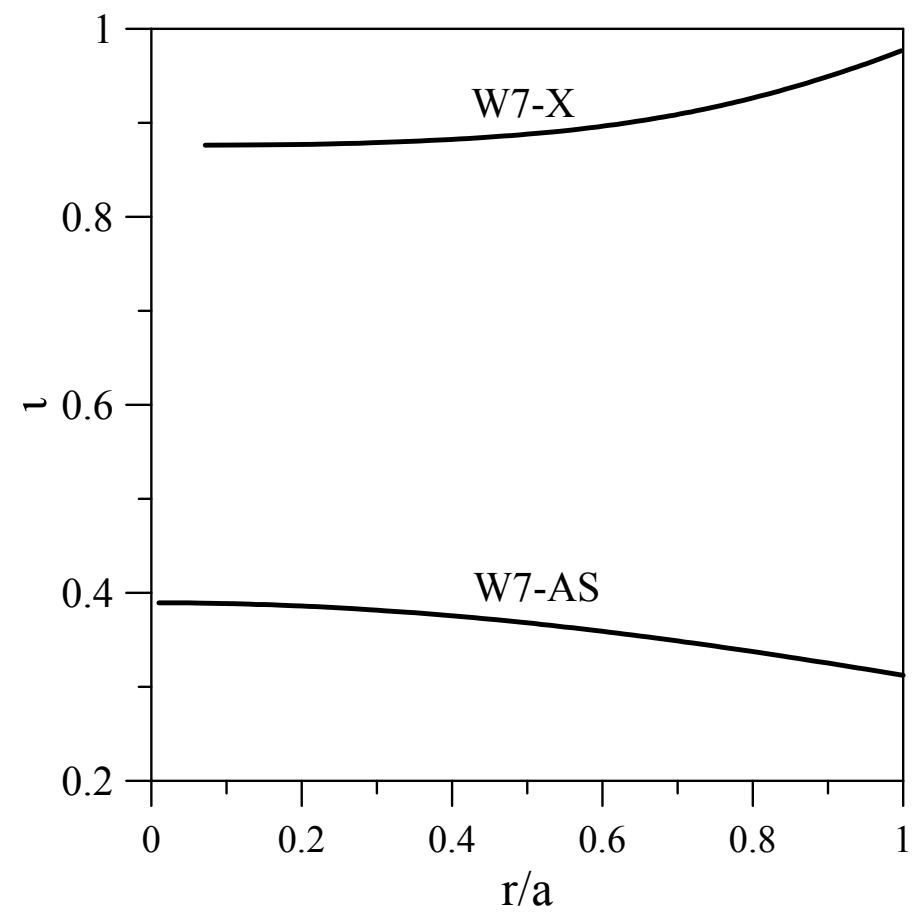

Figure 1. Typical rotational transforms in W7-AS and W7-X.

In this paper we will consider the existence of Alfvénic modes affected by plasma compressibility and having $m=n \neq 0$ in W7-X plasmas with energetic ions produced by NBI.

Note that in GAMs, the dominant Fourier harmonics are known to be $m=n=0$ in the scalar potential of the perturbed electromagnetic field, $\tilde{\Phi}$, and $m=1, n=0$ in the plasma compressibility, $\zeta$, which is coupled to $\tilde{\Phi}$ due to the geodesic curvature of the field lines (This statement is true for relatively low-beta plasmas, $\beta q^{2} \ll 1$ [8], which is the case in $\mathrm{W} 7-\mathrm{X})$. The potential $\tilde{\Phi}$ of the modes with $m=n \neq 0$ is coupled to the compressibility in the same way as in GAMs. Therefore, both the GAM mode and the $m=n \neq 0$ modes can be described by the same equation (however, taking into account the finite orbit width of the energetic ions and / or several coupled harmonics is necessary to determine the GAM radial structure). We will refer to the modes described by this equation as isomon modes, i.e., modes with equal (ISO) MOde Numbers $(m=n)$ of $\tilde{\Phi}$.

The article has the following structure. A basic equation for isomon modes and GAM modes in stellatator plasmas containing energetic passing ions is first derived, and the contribution of the energetic ions being calculated for the $m=n$ case, in section 2 . It is then used to carry out a stability analysis in the local approach in section 3 . The existence of eigenmodes in W7-X is considered and their destabilization in the forthcoming NBI experiments is studied in section 4 . The results obtained are summarized in section 5 . The energetic ions produced by NBI in W7-X are described in Appendix A. In addition, in Appendix A the resonances between the energetic ions (both trapped ions and passing ones) and the modes are considered. The influence of the neoclassical radial electric field on the confinement of locally trapped NBI ions in W7-X is analyzed in Appendix B. 


\section{Basic equations}

In this section we derive equations describing perturbations of Alfvénic character in compressible plasmas with a population of passing energetic ions. We are interested in perturbations with $m=n$ (including the case of $m=n=0$ ), but the restriction $m=n$ will be imposed only on relations associated with the energetic ions.

We proceed from the following equations [9]:

$$
\begin{aligned}
& \nabla \cdot\left(\frac{\omega^{2}}{v_{A}^{2}} \nabla_{\perp} \tilde{\Phi}\right)+\nabla_{\|} \nabla_{\perp}^{2} \nabla_{\|} \tilde{\Phi}-\frac{4 \pi}{c} \nabla \cdot \frac{j_{0 \|}}{B} \tilde{\mathbf{B}}+C^{(s)}=C^{(\alpha)}, \\
& {\left[\omega^{2}\left(1+\beta_{s}\right)+c_{s}^{2} \nabla_{\|}^{2}\right] \tilde{\zeta}=\frac{2 i c \omega}{B^{2}}(\mathbf{B} \times \mathcal{K}) \cdot \nabla \widetilde{\Phi},}
\end{aligned}
$$

where

$$
C^{(s)}=\frac{2 i \omega}{c} \nabla \cdot\left(\beta_{s} \mathbf{B} \times \mathcal{K} \tilde{\zeta}\right)
$$

and

$$
C^{(\alpha)}=\frac{4 \pi i \omega}{c^{2}} \nabla \cdot \tilde{\mathbf{j}}_{\perp}^{(\alpha)}
$$

$\zeta$ is defined by $\zeta=\nabla \cdot \boldsymbol{\xi}, \boldsymbol{\xi}$ is the plasma displacement, a tilde labels perturbed quantities, $\omega$ is the mode frequency, $\mathbf{B}$ is the equilibrium magnetic field, $j_{0 \|}$ is the equilibrium longitudinal plasma current, $\tilde{\mathbf{B}}=-(i c / \omega) \nabla \times\left[\left(\nabla_{\|} \tilde{\Phi}\right) \mathbf{b}\right], \mathbf{b}=\mathbf{B} / B, \nabla_{\|}=\mathbf{b} \cdot \nabla$, $\nabla_{\perp}=\nabla-\mathbf{b} \nabla_{\|}, \mathcal{K}=(\mathbf{b} \cdot \nabla) \mathbf{b}$ is the field line curvature, $\beta_{s}=c_{s}^{2} / v_{A}^{2}, \tilde{\mathbf{j}}^{(\alpha)}$ is the perturbed current of the energetic particles. The first of these equations - equation (1) for the scalar potential of the electromagnetic field coupled to the plasma compressibility due to the field line curvature - is actually the quasineutrality equation $\nabla \cdot \tilde{\mathbf{j}}=0$, with $\tilde{\mathbf{j}}=\tilde{\mathbf{j}}{ }^{(p)}+\tilde{\mathbf{j}}^{(\alpha)}$, $\tilde{\mathbf{j}}^{(p)}$ is the bulk-plasma current, $\tilde{\mathbf{j}}^{(\alpha)}$ is the energetic-ion current. This equation is often used to study Alfvén eigenmodes. The second equation - the equation for $\zeta$ - describes sound waves in the absence of curvature, but includes the curvature coupling of $\zeta$ with $\Phi$. The presence of energetic ions is described by the RHS term in equation (1), $C^{(\alpha)}$, and effects of compressibility in this equation are described by $C^{(s)}$. In equation (2) the inhomogeneity of the magnetic field is neglected except in the term responsible for the coupling of $\zeta$ and $\Phi$. In all equations, the subscripts $\perp$ and $\|$ label magnitudes across and along the equilibrium magnetic field, respectively.

To obtain (1) the equation $\nabla \cdot \tilde{\mathbf{j}}=0$ was expressed as

$$
\nabla \cdot \tilde{j}_{\|} \mathbf{b}+\nabla \cdot \tilde{\mathbf{j}}_{\perp}=0
$$

where $\tilde{j}_{\|}=\tilde{j}_{\|}^{(p)}+\tilde{j}_{\|}^{(\alpha)}$ and $\tilde{j}_{\|}=c \mathbf{b} \cdot(\nabla \times \tilde{\mathbf{B}}) /(4 \pi), \tilde{\mathbf{j}}_{\perp}=\tilde{\mathbf{j}}_{\perp}^{M H D}+\tilde{\mathbf{j}}_{\perp}^{(\alpha)}, \tilde{\mathbf{j}}_{\perp}^{M H D}$ is the current determined by the ideal MHD equations.

Let us take the Fourier transform of the magnetic field (B), the field line curvature $(\mathcal{K})$, and all perturbed quantities $(\tilde{X})$ :

$$
\begin{aligned}
& B=\bar{B}\left(1+\frac{1}{2} \sum_{\mu, \nu} \epsilon_{\mu \nu} e^{i \mu \vartheta-i \nu N \varphi}\right), \\
& \mathcal{K}=\sum_{\mu, \nu} \mathcal{K}_{\mu, \nu}(r) e^{i \mu \vartheta-i N \nu \varphi},
\end{aligned}
$$




$$
\tilde{X}=\sum_{m, n} X_{m, n}(r) e^{i m \vartheta-i n \varphi-i \omega t}
$$

where $\bar{B}$ is the magnetic field at the magnetic axis, the radial coordinate $r$ is defined by $\psi=\bar{B} r^{2} / 2, \psi$ is the toroidal magnetic flux, $\vartheta$ and $\varphi$ are the poloidal and toroidal Boozer angles, respectively, $N$ is the number of the equilibrium field periods. Then, following the approach of Ref. [5], we obtain an equation similar to equation (42) of Ref. [5], but with an extra term describing the contribution of energetic ions. Restricting ourselves to an approximation in which the equilibrium magnetic field contains only harmonics with $\mu=1, \nu=0$, we have:

$$
\begin{aligned}
& \frac{1}{r} \frac{d}{d r} r \delta_{0}\left(\frac{\omega^{2}-\omega_{G 1}^{2}}{v_{A}^{2}}-k_{m n}^{2}\right) \frac{d \Phi_{m, n}}{d r} \\
& -\left[\frac{m^{2} \delta_{0}}{r^{2}}\left(\frac{\omega^{2}-\omega_{G 1}^{2} r^{2} \epsilon_{t}^{\prime 2} / \epsilon_{t}^{2}}{v_{A}^{2}}-k_{m n}^{2}\right)+\frac{k_{m n}}{r}\left(r \delta_{0} k_{m n}^{\prime}\right)^{\prime}\right] \Phi_{m, n} \\
& -\frac{4 \pi i \omega}{c^{2}} B_{m n}^{r} \frac{d}{d r} \frac{j_{0 \|}}{B}=\frac{4 \pi i \omega}{c^{2}}\left(\nabla \cdot \tilde{\mathbf{j}}_{\perp}^{\alpha}\right)_{m, n},
\end{aligned}
$$

where

$$
\omega_{G 1}^{2}=\tilde{\epsilon}^{2} \frac{c_{s}^{2}}{R^{2}} \sum_{l= \pm 1}\left(\frac{\omega^{2}}{\omega^{2}-k_{m+l, n}^{2} c_{s}^{2}}\right),
$$

the potential $\tilde{\Phi}$ is related to the perturbed electric field by $\tilde{\mathbf{E}}=-\nabla_{\perp} \tilde{\Phi}, k_{m n} \equiv k_{\|}(m, n)=$ $(m \iota-n) / R, \delta_{0} \gtrsim 1$ is determined by the plasma shaping (see Ref. [10]), $\tilde{\epsilon}^{2}=\epsilon_{t}^{2} /\left(\delta_{0} \epsilon^{2}\right)$, $\epsilon_{t}=-\epsilon_{1,0}, \epsilon=r / R$, prime denotes the radial derivative.

It follows from equation (9) that the local Alfvén resonance (i.e., Alfvén continuum) in the absence of energetic particles is described by

$$
\omega^{2}=\omega_{G 1}^{2}+k_{m n}^{2} v_{A}^{2}
$$

In particular, when $m=n=0$, this equation has a solution

$$
\omega^{2}=2 \tilde{\epsilon}^{2} \frac{c_{s}^{2}}{R^{2}}(1+\mathcal{S})
$$

where the sound parameter is defined by $\mathcal{S}=\iota^{2} /\left(2 \tilde{\epsilon}^{2}\right)$ [5]. Equation (12) represents the GAM frequency, $\omega_{G A M}$, when $S \ll 1$. In W7-X $\mathcal{S} \gtrsim 1$, whence equation (12) reads:

$$
\omega^{2}=\omega_{s}^{2}\left(1+\mathcal{S}^{-1}\right) \sim \omega_{s}^{2} .
$$

When $m=n \neq 0$, equation (11) has the following simple solution for frequencies well above the sound frequency: $\omega^{2}=m^{2}(\Delta \iota)^{2} v_{A}^{2} / R^{2}$.

The presence of energetic ions affects the continuum and leads to $\operatorname{Im} \omega \neq 0$ when these ions interact resonantly with the perturbation.

As shown in Appendix A, both trapped and passing energetic ions will be produced in the first NBI experiments on W7-X. In the plasma core, ions that are born passing will constitute a majority. For this reason, and because of some uncertainty concerning the confinement of trapped energetic ions, we only consider the effect of passing particles on plasma stability. 

$[6,11]$ :

In general, the resonance condition for passing particles can be written as follows

$$
\omega-[(m+\mu) \iota-(n+\nu N)] \omega_{\varphi}=0,
$$

where $\omega_{\varphi}$ is the frequency of the particle motion in the toroidal direction, which when $m=n$ reduces to

$$
\omega=[-m \Delta \iota+\mu \iota-\nu N)] \omega_{\varphi}
$$

We assume that $m \Delta \iota \ll 1$, which for W7-X yields $m \ll 10$. In addition, we assume that $\omega \ll N \omega_{\varphi}$. Then resonances with $\nu \neq 0$ can thus be neglected. Note that the restriction $\omega \ll N \omega_{\varphi}$ is fulfilled for injected passing ions in W7-X (where $N=5$ ) when the mode frequency is less than $100 \mathrm{kHz}$. For instance, for $60-\mathrm{keV}$ deuterons and $55-\mathrm{keV}$ protons it becomes $\omega \ll 350 \iota \chi \mathrm{kHz}\left(\chi \equiv v_{\|} / v\right)$ and $\omega \ll 470 \iota \chi \mathrm{kHz}$, respectively. This condition does not contradict the condition $\omega>\omega_{s}$ (e.g., $\omega_{s}=20 \mathrm{kHz}$ in a deuterium plasma with temperature $T=4 \mathrm{keV}, \iota=0.87)$. Taking this into account and the fact that in W7-X the magnetic-field Fourier harmonics with $\mu>1$ are small, we only need to consider the interaction of the beam ions and the mode through the resonance

$$
\omega+(m \Delta \iota \pm \iota) \omega_{\varphi}=0 .
$$

As seen from equation (9), the energetic ions contribute through their perturbed current, which we write as

$$
\tilde{\mathbf{j}}_{\perp}^{(\alpha)}=e_{\alpha} \int d^{3} v \mathbf{v}_{D} \tilde{f}
$$

where $\tilde{f}$ is the perturbed distribution function of the energetic ions, $\mathbf{v}_{D}=\left(\mathfrak{w}^{2} / \omega_{B \alpha}\right)(\mathbf{b} \times$ $\mathcal{K})$ the velocity of the particle drift motion, $\mathcal{K} \approx B^{-1} \nabla_{\perp} B, \mathbb{W}^{2}=\left(v_{\|}^{2}+0.5 v_{\perp}^{2}\right) \approx$ const, and $\omega_{B \alpha}=e_{\alpha} \bar{B} /\left(M_{\alpha} c\right)$ is the gyrofrequency, $e$ and $M$ are the electric charge and the particle mass, respectively, the subscript " $\alpha$ " labels energetic ions. It follows from (17) that

$$
\begin{aligned}
& j_{r(m n)}^{(\alpha)}=\frac{e_{\alpha} \epsilon_{t}}{2 i \omega_{B \alpha} r} \int d^{3} v w^{2} \sum_{l= \pm 1} l f_{m+l, n}, \\
& j_{\vartheta(m n)}^{(\alpha)}=-\frac{e_{\alpha} \epsilon_{t}^{\prime}}{2 \omega_{B \alpha} r} \int d^{3} v w^{2} \sum_{l= \pm 1} f_{m+l, n} .
\end{aligned}
$$

The perturbed distribution function can be written as [12]

$$
\tilde{f}=-c \hat{\Pi} F \int_{-\infty}^{t} d \tau \frac{W^{2}}{B}(\mathbf{b} \times \mathcal{K}) \cdot \tilde{\mathbf{E}}-\frac{c R}{i \omega} \frac{\partial F}{\partial J} \tilde{E}_{\varphi}
$$

where $F$ is the equilibrium distribution function of the energetic ions, the operator $\hat{\Pi}$ in variables $\mathcal{E}, \mu_{p}, J$ (the particle energy, the magnetic moment, and the canonical angular momentum, $J=\psi_{p}-R v_{\varphi} B / \omega_{B}$, with $\psi_{p}$ the poloidal magnetic flux) is

$$
\frac{1}{M} \hat{\Pi}=\frac{\partial}{\partial \mathcal{E}}+\frac{c n}{e \omega} \frac{\partial}{\partial J} .
$$

One can see that the Fourier harmonics of $[(\mathbf{b} \times \mathcal{K}) \cdot \tilde{\mathbf{E}}]$ are

$$
[(\mathbf{b} \times \mathcal{K}) \cdot \tilde{\mathbf{E}}]_{m n}=-\frac{1}{2 r} \sum_{l= \pm 1}\left[i l \epsilon_{t} E_{r(m+l, n)}+r \epsilon_{t}^{\prime} E_{\vartheta(m+l, n)}\right]
$$


and therefore,

$$
f_{m n}=-c \hat{\Pi} F \frac{w^{2}}{2 r B} \sum_{l= \pm 1}\left[\frac{l \epsilon_{t} E_{r(m+l, n)}-i r \epsilon_{l}^{\prime} E_{\vartheta(m+l, n)}}{\omega-k_{m n} v_{\|}}\right]-\frac{c R}{i \omega} \frac{\partial F}{\partial J} \tilde{E}_{\varphi(m n)} .
$$

Using (18), (19), (23), $\tilde{\mathbf{E}}=-\nabla_{\perp} \tilde{\Phi}$, and neglecting the coupling between Fourier harmonics, we can write the transverse current components as follows:

$$
\begin{aligned}
& j_{r(m n)}=\frac{i e_{\alpha} c \epsilon_{t}}{4 \omega_{B \alpha} B r^{2}} \sum_{l= \pm 1} \int d^{3} v w^{4} \hat{\Pi} F \frac{\epsilon_{t} \Phi_{m, n}^{\prime}-l m \epsilon_{t}^{\prime} \Phi_{m, n}}{\omega-k_{m+l, n} v_{\|}} \\
& j_{\vartheta(m n)}=\frac{e_{\alpha} c \epsilon_{t}^{\prime}}{4 \omega_{B \alpha} B r} \sum_{l= \pm 1} \int d^{3} v w^{4} \hat{\Pi} F \frac{l \epsilon_{t} \Phi_{m, n}^{\prime}-m \epsilon_{t}^{\prime} \Phi_{m, n}}{\omega-k_{m+l, n} v_{\|}}
\end{aligned}
$$

where $\hat{\Pi}$ in the $(r, v, \chi)$ variables (which are approximately constants of motion for wellpassing particles) is

$$
\hat{\Pi}=\frac{1}{v} \frac{\partial}{\partial v}+\frac{1-\chi^{2}}{v^{2} \chi} \frac{\partial}{\partial \chi}+\left(\frac{R}{v_{\|}}+\frac{n}{\omega}\right) \frac{q}{\omega_{B \alpha}} \frac{1}{r} \frac{\partial}{\partial r} .
$$

In order to calculate the integrals in (24) and (25) we need to specify $F$, which we take as an anisotropic slowing-down distribution

$$
F=\frac{2 n_{\alpha}(r)}{\pi\left(1+\chi_{\alpha}^{2}\right)} \delta\left(\chi-\chi_{\alpha}\right) \frac{\eta\left(v_{\alpha}-v\right)}{v^{3}},
$$

where $\eta\left(v_{\alpha}-v\right)$ is the Heaviside step function, the particle density is defined by $n_{\alpha}=p_{\alpha} / \mathcal{E}_{\alpha}, \mathcal{E}_{\alpha}=0.5 M_{\alpha} v_{\alpha}^{2}, p_{\alpha}$ is the energetic ion pressure defined by $p_{\alpha}=0.5\left(p_{\|}+p_{\perp}\right)$, $p_{\|}=\int d^{3} v v_{\|}^{2} F, p_{\perp}=0.5 \int d^{3} v v_{\perp}^{2} F$, and we take $M_{\alpha}=M_{i}, M_{i}$ is bulk-ion mass. Equation (27) implies that the ion energy is sufficiently high, $\mathcal{E} \gg\left(M_{i} / M_{e}\right)^{1 / 3} T\left(M_{e}\right.$ is the electron mass) so that Coulomb collisions mainly slow down the fast ions without much pitch-angle scattering.

By means of equations (24) - (27), we obtain the energetic particle term in (9) in the form:

$$
\begin{aligned}
& C_{m n}^{(\alpha)} \equiv \frac{4 \pi i \omega}{c^{2}}\left(\nabla \cdot \tilde{\mathbf{j}}_{\perp}^{\alpha}\right)_{m, n} \\
& =\sum_{l= \pm 1} \frac{1}{r}\left(\frac{\partial}{\partial r} r \frac{\epsilon_{t}^{2}}{\epsilon^{2}}+\operatorname{lm} \frac{r \epsilon_{t}^{\prime} \epsilon_{t}}{\epsilon^{2}}\right) \frac{\omega^{2}}{v_{A}^{2}} N_{\alpha} \alpha_{l}(\omega)\left(\frac{\partial \Phi_{m, n}}{\partial r}-\operatorname{lm} \frac{\epsilon_{t}^{\prime}}{\epsilon_{t}} \Phi_{m, n}\right)
\end{aligned}
$$

where

$$
\begin{aligned}
& \alpha_{l}(\omega)=\psi\left(\chi_{\alpha}^{2}\right)\left[\frac{\sigma_{\chi} \omega_{t}}{\xi_{l} \omega}+\frac{1}{\xi_{l}^{2}} \ln \left(1-\sigma_{\chi} \xi_{l} \frac{\omega_{t}}{\omega}\right)\right]+\frac{\left(1+\chi_{\alpha}^{-2}\right)^{2} \omega_{t}^{2}}{\omega^{2}-\sigma_{\chi} \xi_{l} \omega \omega_{t}} \\
& -\left|\chi_{\alpha}\right| \mathcal{N}\left(\chi_{\alpha}^{2}\right)\left\{\frac{n \sigma_{\chi}}{3 \iota \xi_{l}} \frac{\omega_{t}^{2}}{\omega^{2}}+\frac{(m+l)}{\xi_{l}^{2}}\left[\frac{\omega_{t}}{2 \omega}+\frac{\sigma_{\chi}}{\xi_{l}}+\frac{\omega}{\xi_{l}^{2} \omega_{t}} \ln \left(1-\sigma_{\chi} \xi_{l} \frac{\omega_{t}}{\omega}\right)\right]\right\} \\
& N_{\alpha}=\frac{n_{\alpha}}{n_{i}} \frac{1}{4 \iota^{2}\left(1+\chi_{\alpha}^{2}\right)} \\
& \psi\left(\chi_{\alpha}^{2}\right)=3 \chi_{\alpha}^{-4}-2 \chi_{\alpha}^{-2}-5 \\
& \mathcal{N}\left(\chi_{\alpha}^{2}\right)=-\left(1+\chi_{\alpha}^{-2}\right)^{2} \frac{R \rho_{\alpha}}{r \iota} \frac{d \ln n_{\alpha}}{d r}
\end{aligned}
$$


$\xi_{l}=k_{m+l, n} R / \iota, \omega_{t}=v_{\alpha}\left|\chi_{\alpha}\right| \iota / R$ is the particle transit frequency, $\rho_{\alpha}=v_{\alpha} / \omega_{B \alpha}$, $\omega_{B \alpha}=e_{\alpha} B /\left(M_{\alpha} c\right), \sigma_{\chi}=\operatorname{sgn} \chi_{\alpha}, \ln \left(1-\sigma_{\chi} \xi_{l} \omega_{t} / \omega\right)=\ln \left|1-\sigma_{\chi} \xi_{l} \omega_{t} / \omega\right|+i \pi$ for $\sigma_{\chi} \xi_{l} \omega_{t} / \omega>1$. Substituting equation (28) into equation (9) and assuming $j_{0}=0$ we obtain

$$
\begin{aligned}
& \frac{1}{r} \frac{d}{d r} r \delta_{0}\left(\frac{\omega^{2}-\omega_{G 1}^{2}-\omega_{\alpha}^{2}}{v_{A}^{2}}-k_{m n}^{2}\right) \frac{d \Phi_{m, n}}{d r}-\left\{\frac { m ^ { 2 } \delta _ { 0 } } { r ^ { 2 } v _ { A } ^ { 2 } } \left[\omega^{2}-\frac{r^{2} \epsilon_{t}^{\prime 2}}{\epsilon_{t}^{2}}\left(\omega_{G 1}^{2}+\omega_{\alpha}^{2}\right)\right.\right. \\
& \left.\left.-\frac{r v_{A}^{2}}{m \delta_{0}}\left(\frac{\delta_{0} r \epsilon_{t}^{\prime}}{\epsilon_{t}} \frac{\sum_{l} l \omega_{\alpha, l}^{2}}{v_{A}^{2}}\right)^{\prime}-k_{m n}^{2} v_{A}^{2}\right]+\frac{k_{m n}}{r}\left(r \delta_{0} k_{m n}^{\prime}\right)^{\prime}\right\} \Phi_{m, n}=0
\end{aligned}
$$

where

$$
\omega_{\alpha}^{2}=\sum_{l= \pm 1} \omega_{\alpha, l}^{2}, \quad \omega_{\alpha, l}^{2}=\tilde{\epsilon}^{2} \omega^{2} N_{\alpha} \alpha_{l}(\omega)
$$

This equation is valid for modes with $m=n$, including the case $m=n=0$ (GAM modes) in both stellarators and tokamaks. In tokamaks $\epsilon_{t}=\epsilon$, whereas in stellarators $\epsilon_{t} \neq \epsilon$; in particular, $\epsilon / \epsilon_{t} \sim 2.4$ in W7-X. When $m=n=0$, equation (29) for $\alpha_{l}(\omega)$ is reduced, giving the following expression for $\sum_{l} \alpha_{l}(\omega)$ :

$$
\begin{aligned}
& \sum_{l= \pm 1} \alpha_{l}(\omega)=2\left(1+\chi_{\alpha}^{-2}\right)^{2} \frac{\omega_{t}^{2}}{\omega^{2}-\omega_{t}^{2}}+\psi\left(\chi_{\alpha}^{2}\right) \ln \left(1-\frac{\omega_{t}^{2}}{\omega^{2}}\right) \\
& -\chi_{\alpha} \mathcal{N}\left(\chi_{\alpha}^{2}\right)\left(2+\frac{\omega}{\omega_{t}} \ln \frac{\omega-\omega_{t}}{\omega+\omega_{t}}\right) .
\end{aligned}
$$

Equations (34), (35) agree with the corresponding result for tokamaks of Ref. [9]. The difference of a factor of two is associated with different definitions of $n_{\alpha}$ here and in Ref. [9], and the presence of the factor $\tilde{\epsilon}^{2}$ in equation (34) is due to the plasma elongation and the fact that $\epsilon_{t} \neq \epsilon$ in stellarators.

\section{Stability analysis in local approach}

It follows from equation (33) that the Alfvén resonance equation (11) in the presence of energetic ions should be replaced by

$$
\omega^{2}-\omega_{G 1}^{2}-k_{m n}^{2} v_{A}^{2}=\omega_{\alpha}^{2} .
$$

This can be considered as an eigenvalue problem of the form $\Lambda(\omega)=\lambda(\omega)$, with $\Lambda=\omega^{2}-\omega_{G 1}^{2}(\omega)-k_{m n}^{2} v_{A}^{2}$ and $\lambda=\omega_{\alpha}^{2}$. Because the number of energetic particles is small, it can be solved perturbatively. Taking $\omega=\omega_{0}+\Delta$, where $\Delta \ll \omega_{0}$, we obtain:

$$
\Lambda\left(\omega_{0}\right)=0, \quad \Delta=\frac{\lambda\left(\omega_{0}\right)}{\partial \Lambda(\omega) /\left.\partial \omega\right|_{\omega_{0}}} .
$$

In particular, when the mode frequency well exceeds the sound frequency, so that $\omega_{G 1}^{2}=2 \tilde{\epsilon}^{2} c_{s}^{2} / R^{2} \equiv \omega_{G}^{2}$ is the reduced GAM frequency $\left[\omega_{G A M}(\mathcal{S}=0)=\omega_{G}\right]$, we have:

$$
\omega^{2}=k_{m n}^{2} v_{A}^{2}+\omega_{G}^{2}, \quad \frac{\gamma}{\omega}=\frac{1}{2} \tilde{\epsilon}^{2} N_{\alpha} \operatorname{Im} \alpha,
$$

where $\gamma=\operatorname{Im} \Delta, \alpha=\sum_{l= \pm 1} \alpha_{l}$, and the subscript " 0 " is omitted. When $m=n=0$, the frequency is given by equation (12) and the $\gamma / \omega$ ratio is described by (38) supplemented with the factor $(1+\mathcal{S})^{-1}$. 
Note that $\operatorname{Im} \alpha \neq 0$ provided that

$$
\omega<\omega_{t}\left|\xi_{l}\right|, \quad \xi_{l} \chi_{\alpha}>0,
$$

where $\xi_{l}$ for the $m=n$ modes is $\xi_{l}=l-m \Delta \iota / \iota$. In the considered case of $|m \Delta \iota|<1$ the second inequality in (39) is simplified to

$$
l \chi_{\alpha}>0,
$$

which means that, depending on the sign of $\chi_{\alpha}$, either $l=1$ or $l=-1$ contributes to the sum in $\operatorname{Im} \alpha$.

It follows from equation (38) that energetic-ion-driven instabilities can arise when $\operatorname{Im} \alpha>0$. Both the velocity-space anisotropy and the spatial inhomogeneity of the energetic ions contribute to the growth rate of the instability. Instabilities driven by the velocity-space anisotropy arise when $\chi_{\alpha}^{2}<0.6$, and do not depend on the beam direction.

The spatial inhomogeneity can destabilize the modes when

$$
m+l<0 .
$$

The effect of the inhomogeneity is sensitive to the beam direction and the direction of the mode rotation (the sign of $m$ ), as follows from equations (40), (41). In particular, in the case of GAM modes the inhomogeneity of counter-passing particles $\left(\sigma_{\chi}<0\right)$ is a destabilizing factor, whereas the inhomogeneity of co-passing particles $\left(\sigma_{\chi}\right)$ produces a stabilizing effect. The growth rate of the $m \neq 0$ modes is largest when two inequalities, $m<0$ and $\sigma_{\chi}<0$, are satisfied simultaneously. In this case the inhomogeneity part in the expression for the growth rate is proportional to $(1+|m|) /(1-|m| \Delta \iota / \iota)^{4}$. It dominates when

$$
\left|(m+l) \chi_{\alpha}\right| \mathcal{N}\left(\chi_{\alpha}^{2}\right) \sqrt{\frac{\mathcal{E}_{r e s}}{\mathcal{E}_{\alpha}}} \gg\left|\xi_{l} \psi\left(\chi_{\alpha}^{2}\right)\right| .
$$

These relations are valid when the resonance condition [leading to the first inequality in (39)] is satisfied, i.e., when

$$
\omega=\left|\xi_{l}\right| \omega_{t} \frac{v}{v_{\alpha}} \text {. }
$$

For the frequency satisfying equation (38) this resonance condition has the form:

$$
k_{m n}^{2} v_{A}^{2}\left(1+\frac{\omega_{G}^{2}}{k_{m n}^{2} v_{A}^{2}}\right)=\left(k_{m n}+\frac{l \iota}{R}\right)^{2} v^{2} \chi_{\alpha}^{2} .
$$

In W7-X $v_{\alpha}<v_{A}$ (for instance, $v_{A} / v_{\alpha}=1.7$ when the plasma ions and beam ions are of the same species, the plasma ion density $n_{i}=8 \times 10^{19} \mathrm{~m}^{-3}$ and beam ions (protons) have the energy $55 \mathrm{keV}$ ). Taking this into account and that $v<v_{\alpha}, \chi_{\alpha}<1$, we infer that equation (44) requires $k_{m n}^{2} R^{2} / \iota^{2} \ll 1$, i.e., $[m \Delta \iota / \iota]^{2} \ll 1$ for the modes with $m=n$.

Let us now evaluate the instability growth rate in W7-X. It follows from (29), (38) that the growth rate depends sensitively on the pitch angle $\left(\psi=0\right.$ for $\chi_{\alpha}^{2}=0.6$ and $\psi>0$ is maximum at low $\chi$ ). The pitch angle of passing particles is restricted by the condition $\chi>\left(\sum\left|\epsilon_{\mu \nu}\right|\right)^{1 / 2}$. The main Fourier harmonics $\epsilon_{\mu \nu}$ for the high mirror configuration of W7-X are shown in figure 2 , from which it follows that the minimum reasonable pitch angle of passing particles is $\chi_{\alpha}=0.5$. Using this pitch angle, taking $\tilde{\epsilon}^{2} / \iota^{2}=0.14$, 

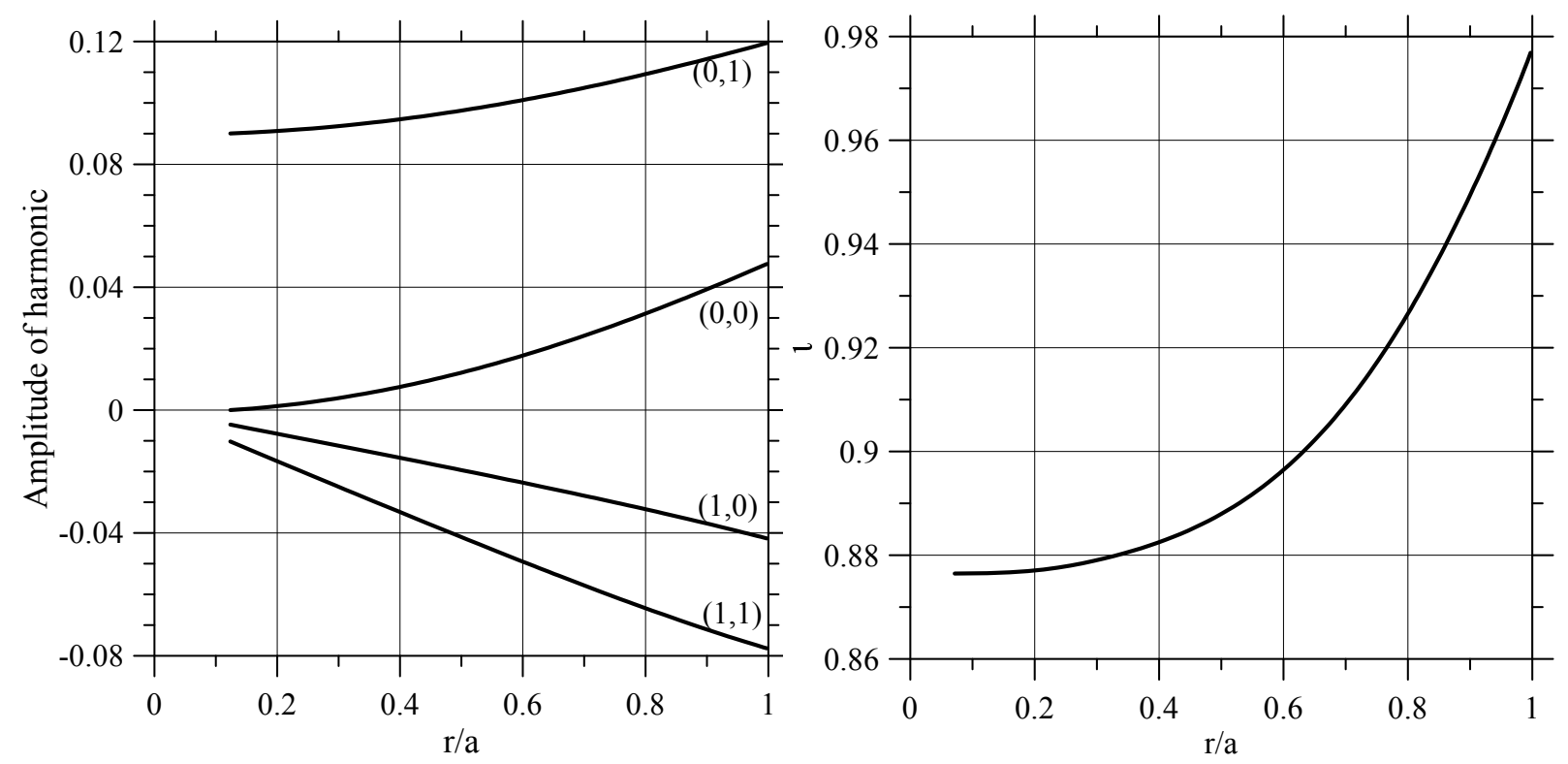

Figure 2. Fourier harmonics of the equilibrium magnetic field and the rotational transform of the field lines for $\beta(0)=0.037$ in the W7-X high-mirror configuration. Here $r$ is the radial flux coordinate, $a$ is the plasma radius.
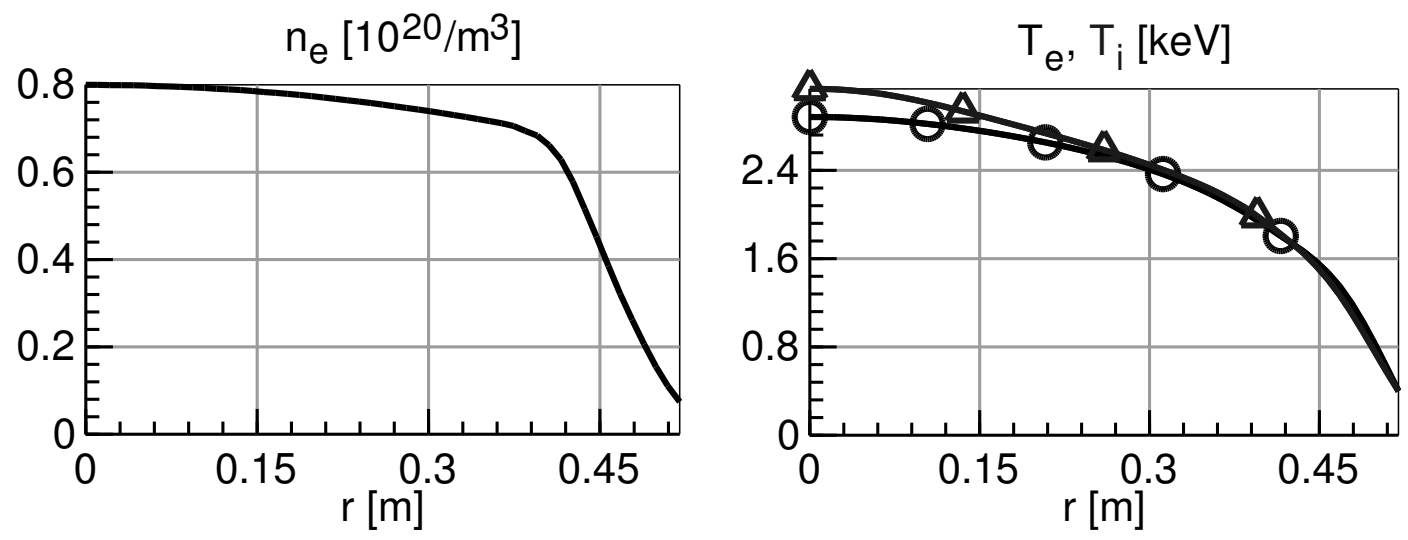

Figure 3. The plasma density and temperature determined by neoclassical transport in W7-X. The subscripts "e" and "i" label electrons and ions, respectively, circles are relevant to electrons and triangles to ions.

and ignoring the contribution of the inhomogeneity of fast ions, we obtain the following estimate for the growth rate:

$$
\frac{\gamma}{\omega}=\frac{\pi}{2} \tilde{\epsilon}^{2} N_{\alpha} \psi \sum_{l= \pm 1} \frac{\eta\left(z_{l}\right)}{\xi_{l}^{2}} \sim \frac{n_{\alpha}}{n_{i}}
$$

where $\eta\left(z_{l}\right)$ is a step function, $z_{l}=\sigma_{\chi} \xi_{l} \omega_{t} / \omega-1$. The plasma inhomogeneity increases this estimate for the growth rate when the condition (41) is satisfied.

The magnitude of $n_{\alpha} / n_{i}$ can be evaluated as follows. According to our definition of 
the fast-ion density, $n_{\alpha}=\left(p_{\perp}+p_{\|}\right)\left(2 \mathcal{E}_{\alpha}\right)$, which leads to

$$
n_{\alpha}=\frac{1+\chi_{\alpha}^{2}}{2} \frac{W}{\mathcal{E}_{\alpha}}
$$

Here $W=\int d^{3} v\left(M v^{2} / 2\right) F$ is the density of the fast ion energy content, which is determined by the injected power density, $P_{i n j}$, as follows: $W=P_{i n j} \tau_{\mathcal{E}}$, with $\tau_{\mathcal{E}}$ the particle energy loss caused by collisional slowing down. Assuming $P_{i n j}(r)=P_{i n j}(0) \eta_{i n j}(r)$ and using the identity $\int d^{3} x P_{i n j}=\bar{P}_{i n j} V_{p}$, where $\bar{P}_{i n j}$ is the average injected power and $V_{p}$ is the plasma volume, we can eliminate $P_{i n j}(0)$ and obtain

$$
n_{\alpha}=\frac{1+\chi_{\alpha}^{2}}{2} \frac{\bar{P}_{i n j}}{\mathcal{E}_{\alpha}} \frac{V_{p}}{\int d^{3} x \eta_{i n j}} \tau_{\mathcal{E}} \eta_{i n j} .
$$

For instance, when $\eta_{i n j}=\left(1-r^{2} / a^{2}\right)^{\nu_{n}}$, equation (47) becomes

$$
n_{\alpha}=\frac{1+\chi_{\alpha}^{2}}{2} \frac{\mathcal{P}_{i n j}}{V_{p} \mathcal{E}_{\alpha}}\left(\nu_{n}+1\right) \tau_{\mathcal{E}} \eta_{i n j}
$$

where $\mathcal{P}_{i n j}$ is the total injected power.

In the first NBI experiments on W7-X, the injected power will be up to $6.84 \mathrm{MW}$, and the maximum energy of injected particles (protons) will be $55 \mathrm{keV}$. The expected plasma parameters in the core region are $T_{e}=2.9 \mathrm{keV}, n_{e}=8 \times 10^{19} \mathrm{~m}^{-3}$, and the plasma volume $V_{p}=30 \mathrm{~m}^{3}$, see figure 3 . With these parameters and $n_{i}=n_{e}$, we obtain

$$
\frac{n_{\alpha}(0)}{n_{i}(0)}=0.9\left(\nu_{n}+1\right) \frac{1+\chi_{\alpha}^{2}}{2} \% \text {. }
$$

The NBI energy deposition is rather peaked, $\nu_{n} \gg 1$. For $\nu_{n}=5, \chi_{\alpha}=0.6$ we obtain: $n_{\alpha}(0) / n_{i}(0)=3.7 \%$.

Note that strong transient isomon instabilities, lasting for $\Delta t \ll \tau_{\mathcal{E}}$ and involving resonant particles with different pitch angles, may arise immediately after the NBI source is switched on. This conclusion is based on the following. Before a monotonically decreasing energy distribution of the injected ions will be formed [e.g., the distribution given by equation (27)], their distribution function will evolve in time, with $\partial F / \partial \mathcal{E}>0$ in a region ranging from the birth energy down to low energies. This evolution is similar to that shown in figure 8 of reference [13] for fusion produced alpha particles. The presence of a region with $\partial F / \partial \mathcal{E}>0$ is an additional factor driving the instability.

Thus, it appears that the growth rate of isomon instabilities in $\mathrm{W} 7-\mathrm{X}$ can be rather large unless the drive from fast ions is overcome by damping mechanisms in the background plasma.

\section{Eigenmode analysis}

One can expect that an isomon mode will exist when a corresponding continuum branch has an extremum. For this to be the case, the following equation should be satisfied:

$$
\left(k_{m n}^{2} v_{A}^{2}\right)^{\prime}+\left(\omega_{G 1}^{2}\right)^{\prime}+\left(\omega_{\alpha}^{2}\right)^{\prime}=0 .
$$

This equation can be written as follows:

$$
2 \iota^{\prime}-\frac{\eta_{n} R^{2}}{\Delta \iota m^{2} v_{A 0}^{2}}\left[\left(\omega_{G 1}^{2}\right)^{\prime}+\left(\omega_{\alpha}^{2}\right)^{\prime}\right]=-\Delta \iota \frac{\eta_{n}^{\prime}}{\eta_{n}},
$$


where $\eta_{n}=n_{i}(x) / n_{i}(0), x=r / a, a$ is the plasma radius. Let us assume that (i) the radial profiles of the plasma parameters and fast ions are decreasing functions of the minor radius; (ii) $\iota^{\prime}>0$ (which is the case in W7-X and in many other stellarators); and (iii) $\Delta \iota>0$. Then all the terms on both sides of Eq. (51) are positive (when $\operatorname{Im} \omega_{\alpha} \neq 0$, our statement is true for the real part of the fast ion term). Because the RHS consists of a single term associated with the inhomogeneity of the plasma density, equation (51) can be satisfied only in inhomogeneous plasmas. On the other hand, finite magnetic shear, plasma compressibility, and the energetic ions contribute to the LHS of (51).

A simple solution of (51) can be obtained for an incompressible plasma without energetic ions. Let us take

$$
\iota=\iota_{0}+\left(\iota_{a}-\iota_{0}\right) x^{2},
$$

where $\iota_{0}=\iota(0)$ and $\iota_{a}=\iota(a)$. Equation (52) is justified, at least, when the magnetic shear is small in the entire plasma cross section. The plasma density profile is approximated by $\eta_{n}(x)=\left(1-x^{2}\right)^{1 / 2}$. Then, solving (51) for $x^{2}$, we obtain

$$
x_{*}^{2}=\frac{1}{3}\left(4-\frac{1-\iota_{0}}{\iota_{a}-\iota_{0}}\right) \text {. }
$$

In W7-X $\iota_{a}-\iota_{0} \approx 0.1$. In this case, as seen from (53), $x_{*}=1$ at $\iota_{0}=0.9$, and $x_{*} \ll 1$ when $\iota_{0}$ is well below 0.9 . This implies that it is more likely that an extremum exists in the W7$\mathrm{X}$ standard and low- $\iota$ configurations than in the high-iota and high-mirror configurations (where $\iota_{0}$ is somewhat higher). Another conclusion is that the plasma compressibility and the presence of the energetic ions are factors that facilitate the existence of extrema.

However, this does not necessarily mean that the compressibility will lead to discrete modes. The point is that it breaks up the Alfvén continuum, produces the sound branch, and shifts upwards the Alfvén branch located above the sound branch, this shift being dependent on the radius. As a result of even a small shift, the mode frequency may cross a continuum branch [in the $\left(\mathrm{r}, \omega\right.$ plane)] at some point $r_{+}$. Then a discontinuity of the derivative $\Phi^{\prime}(r)$ in $r_{+}$in the numerically calculated mode $\Phi(r)$ appears. Strictly speaking, in this case the eigenmode does not exist because of continuum damping, but it will nevertheless survive if there is a population of energetic ions whose drive exceeds the damping.

The role of the energetic ions is not restricted to mode destabilization. They can also lead to the appearance of an additional continuum branch, as shown in figure 4.

For numerical calculations it is convenient to write equation (33) in dimensionless form. We introduce the dimensionless wave number $\bar{k}_{m n}=k_{m n} R$, the plasma temperature $\Theta(x)=T(x) / T(0), \bar{\omega}=\omega R / c_{s 0}$ and $\bar{\omega}_{t}=\omega_{t} R / c_{s 0}$, with $c_{s 0}=c_{s}(0)$. Then we will have (bars over $k_{\|}, \omega$ and $\omega_{t}$ are omitted):

$$
\begin{aligned}
& \frac{1}{x} \frac{d}{d x} x \delta_{0}\left[\left(\omega^{2}-\omega_{G 1}^{2}-\omega_{\alpha}^{2}\right) \eta_{n} \beta_{s 0}-k_{m n}^{2}\right] \frac{d \Phi_{m, n}}{d x}-\frac{m^{2}}{x^{2}}\left\{\delta _ { 0 } \eta _ { n } \beta _ { s 0 } \left[\omega^{2}-\frac{x^{2} \epsilon_{t}^{\prime 2}}{\epsilon_{t}^{2}}\left(\omega_{G 1}^{2}+\omega_{\alpha}^{2}\right)\right.\right. \\
& \left.\left.-\frac{x}{m \delta_{0} \eta_{n}}\left(\frac{\delta_{0} x \epsilon_{t}^{\prime}}{\epsilon_{t}} \eta_{n} \sum_{l} l \omega_{\alpha, l}^{2}\right)^{\prime}\right]-\delta_{0} k_{m n}^{2}\right\} \Phi_{m, n}-\frac{k_{m n}}{x}\left(x \delta_{0} k_{m n}^{\prime}\right)^{\prime} \Phi_{m, n}=0,
\end{aligned}
$$


where

$$
\begin{aligned}
& \omega_{G 1}^{2}=\tilde{\epsilon}^{2} \Theta \sum_{l= \pm 1} \frac{\omega^{2}}{\omega^{2}-[(m+l) \iota-n]^{2} \Theta}, \\
& \mathcal{N}\left(\chi_{\alpha}\right)=-\left(1+\chi_{\alpha}^{-2}\right)^{2} \frac{R}{a} \frac{\rho_{\alpha}}{a} \frac{1}{\iota x} \frac{d \ln n_{\alpha}}{d x}
\end{aligned}
$$

$\omega_{\alpha}$ and $\omega_{\alpha, l}$ are given by equation $(34), \beta_{s 0}=c_{s}^{2}(0) / v_{A}^{2}(0), k_{m n}=m \iota-n, \xi_{l}=m+l-n / \iota$, $\ln z=\ln |z|+i \pi$ when $\operatorname{Re} z<0$.

Equation (54) was solved for the W7-X high mirror configuration with $\beta_{0}=3.7 \%$ and the standard configuration with $\beta_{0}=6.8 \%$. Plasmas without energetic ions and with NBI ions (with $n_{\alpha}(0) / n_{i}(0)=3 \%$ ) were considered. Some results of these calculations are shown in figures $4-6$.

Figure 4 demonstrates that isomon instabilities with global structures and rather large growth rates can be excited in the high-mirror configuration. Of course, this statement is only true when the destabilizing effect of the energetic ions exceeds the mode damping. To study the damping is however beyond the scope of this paper. Here we restrict ourselves to a brief discussion of this issues.

First of all, we note that the phase velocity with the $k_{\|} R=m(\iota-1)$ of the calculated mode well exceeds the bulk ion thermal velocity, $\left(\omega / k_{\|} v_{T i}\right)^{2} \sim\left(c_{s} / v_{T i}\right)^{2} /(m \Delta \iota)^{-2} \gg 1$. This implies that ion damping due to the plasma displacement across the magnetic field $\left(\xi_{\perp} \sim c \tilde{\Phi} /(r \bar{B} \omega)\right)$, is relatively small. However, one can show that $\xi_{\|} \sim \xi_{\perp}$ for $\omega^{2} \sim 2 c_{s}^{2} / R^{2}$ (we used $\left.\mathcal{K}=(1 / R) \epsilon_{t} / \epsilon\right)$ and $\omega^{2} / k_{s}^{2} c_{s}^{2} \sim 2 c_{s}^{2} / v_{T}^{2}$. Therefore, the damping associated with the plasma displacement along the magnetic field is not negligible and deserves a special study.

A mode resembling the $m=n=1$ rigid kink displacement in tokamaks inside the $q=1$ radius but, in contrast to the tokamak mode, occupying almost the entire plasma cross section, was found in the case of the high mirror configuration with $\iota(r)$ a little bit smaller than that shown in figure 2 , see figure 5 .

Calculations were also carried out for the W7-X standard configuration having a lower $\iota(r)$ than in figure 2, in which case equation (50) is more easily satisfied. This explains why the modes were found even in the absence of energetic ions, see figure 6 . Note that the frequencies of these modes are higher than that of the mode shown in figure 4 and, hence, their Landau damping on ions is smaller.

These results are based on equation (54) obtained with a number of simplifying assumptions. Therefore, assuming that the energetic ions are absent, we carried out calculations with the full ideal MHD code CAS3D [14] and the CKA code [15]. The latter is based on reduced MHD and neglects plasma compressibility. The $m=n=3$ and $m=n=4$ modes calculated by the CAS3D with compressibility switched off and by the CKA code are shown in figure 7 . We observe a good agreement between the results of these codes. Furthermore, the calculated modes are in reasonable agreement with the modes described by equation (54) but calculated for the standard magnetic configuration (see figure 6). This is not surprising because the rotational transform in the standard configuration is only a little bit less than that in the high mirror configuration. On the other hand, the code CAS3D does not find the $m=n=4, m=n=3$ and $m=n=2$ 

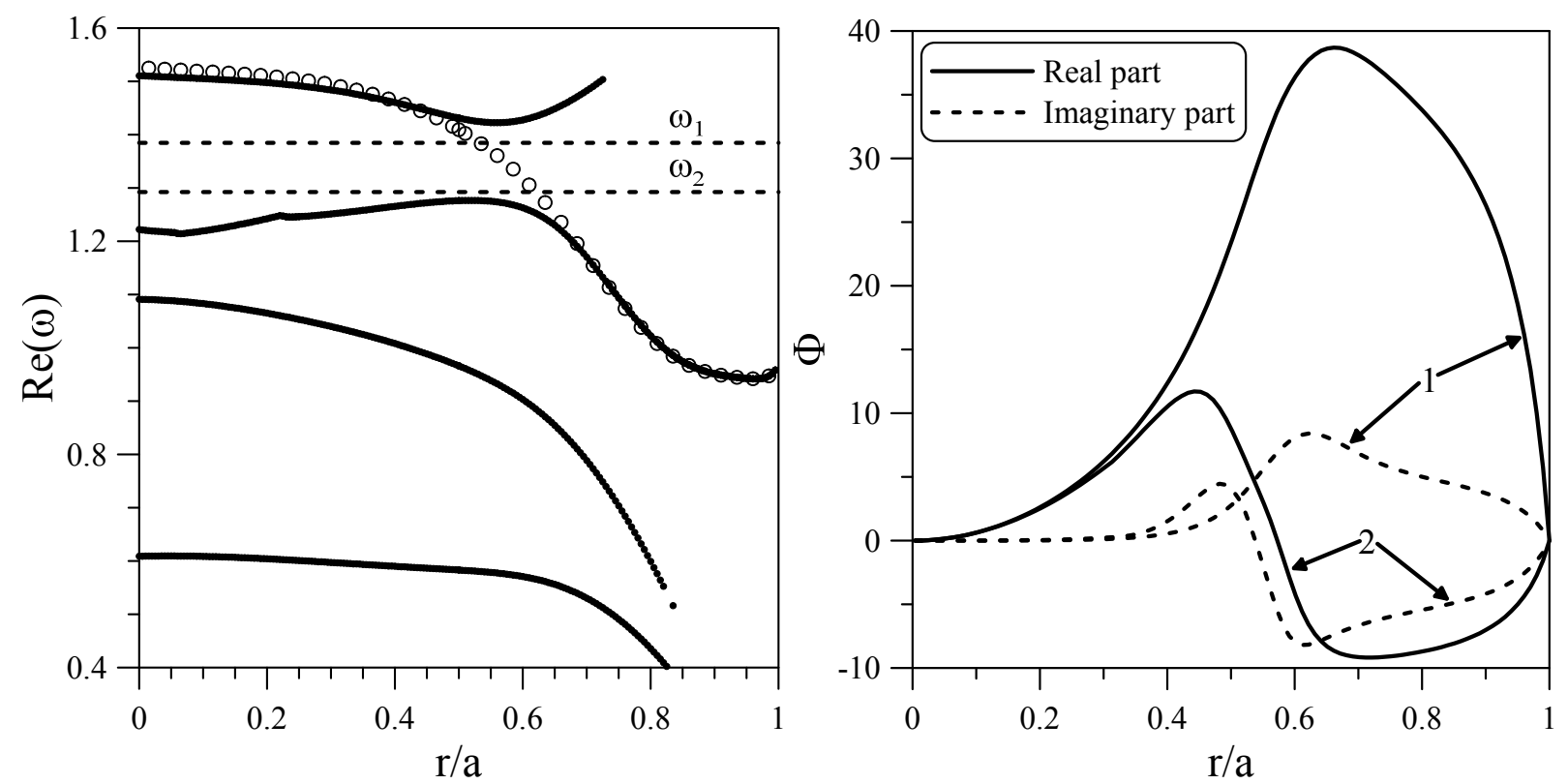

Figure 4. The $m=n=-2$ continuum branches shown by solid lines (left panel) and the modes with $\omega_{1}=1.384$ (approximately $40 \mathrm{kHz}$ at $T(0)=2.9 \mathrm{keV}$ ), $\gamma_{1}=0.01$ and $\omega_{2}=1.292$ (approximately $37 \mathrm{kHz}$ at $T(0)=2.9 \mathrm{keV}$ ), $\gamma_{2}=0.048$ (right panel) in the W7-X high-mirror configuration in the presence of counter-injection of hydrogen with $\chi_{\alpha}=-0.55, n_{\alpha}(0)=0.03$. The dashed lines in the left panel show frequencies of two modes found. They lie in the continuum gap which has arisen to avoid crossing the intersection point of the upper continuum branch described by equation (11) and an energetic-ion-induced branch. The two lower continuum curves depend weakly on the presence of the energetic ions. The numbers "1" and "2" in the right panel label modes with the frequencies $\omega_{1}$ and $\omega_{2}$, respectively.

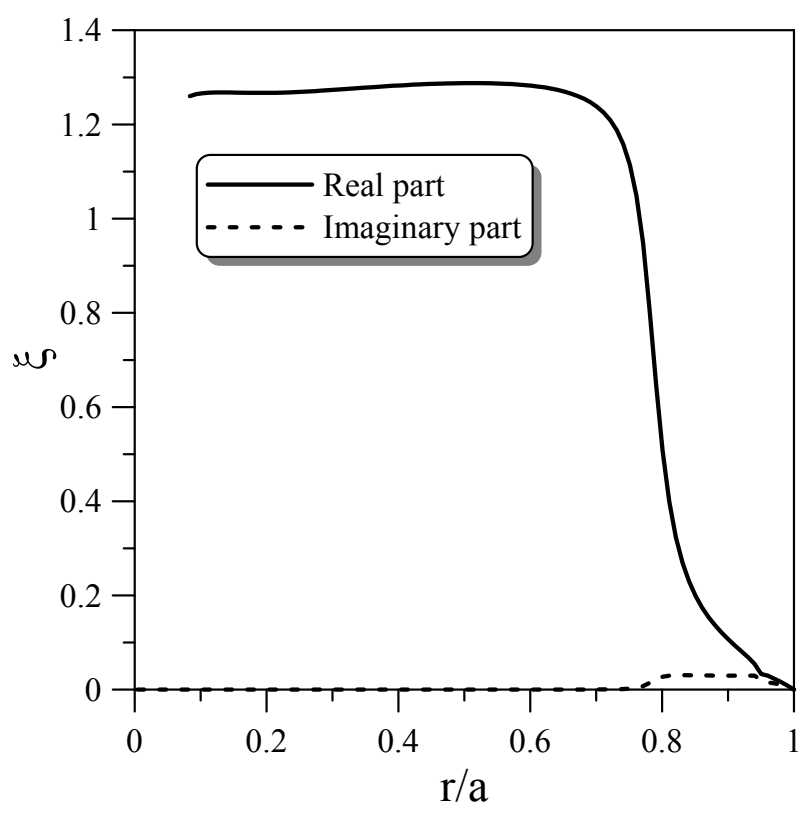

Figure 5. The $m=n=-1$ mode with $\omega=1.821, \gamma=0.001$ in a plasma with similar parameters to those in figure 4 but with $\iota(r)$ everywhere decreased by by 0.015 . 

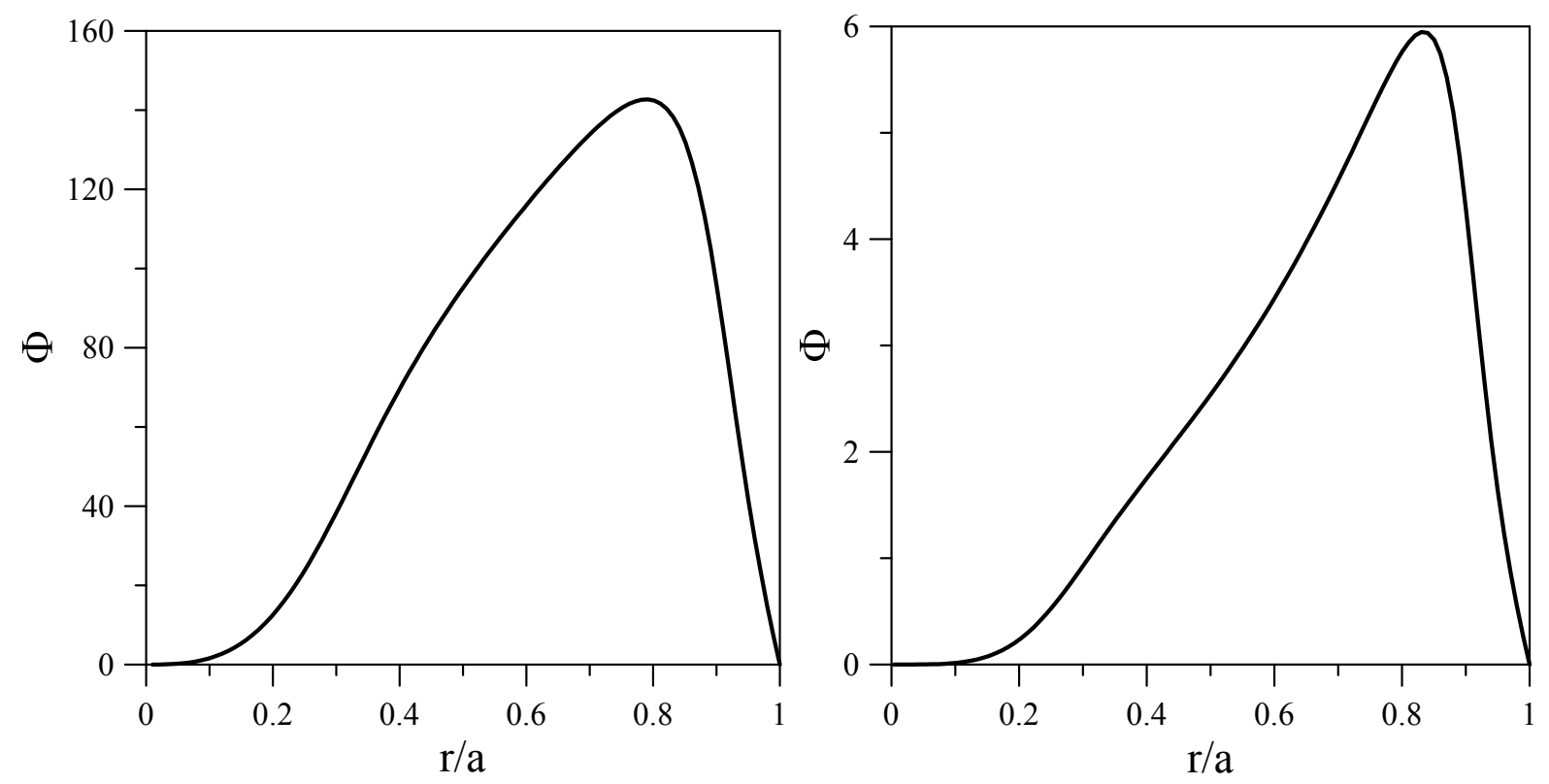

Figure 6. Eigenmode with $|m|=|n|=3, \omega=1.980, \gamma=0$ (left panel) and eigenmode with $|m|=|n|=4, \omega=2.562, \gamma=0$ (right panel) in the W7-X standard configuration without fast particles.

modes in compressible plasmas: The calculated modes have strong spikes at certain radii, which indicates strong continuum damping. A similar picture was obtained by solving equation (54) in the absence of the energetic ions.

Figures 4-7 demonstrate that isomon modes exist and may extend over a large part of the plasma cross section. The plasma compressibility is a factor which may prevent the existence of these modes, whereas the presence of the energetic ions and a decrease of the rotational transform facilitate the mode existence.

\section{Summary and conclusions}

The results of this work can be summarized as follows.

- W7-X is prone to Isomon Modes (IM), which are Alfvénic eigenmodes affected by plasma compressibility and having $m=n$. These modes are sensitive to the magnitude of the rotational transform. In the high-mirror configuration, the IM modes were found to exist in the presence of energetic ions. The same modes were also found in the absence of such ions, but suffer some continuum damping in this case. A very wide $m=n=1$ mode was found, which constitutes a rigid kink displacement of almost the entire plasma cross section. In the standard configuration, where $\iota(r)$ is only slightly less, by $1-2 \%$, eigenmodes of this kind were found even in the absence of the energetic ions.

- The passing NBI ions (with the maximum energy $55-60 \mathrm{keV}$ ) tend to destabilize the IM modes with $m \ll \iota / \Delta \iota \approx 10$ in W7-X. The instability drive from the fast ions corresponds to a growth rate of about $\gamma / \omega=10^{-2}-10^{-3}$ in the first planned NBI experiments. Whether the mode will actually be unstable depends, of course, on the damping, which we have not calculated.

- These results are based on the mode equation which takes into account the plasma 

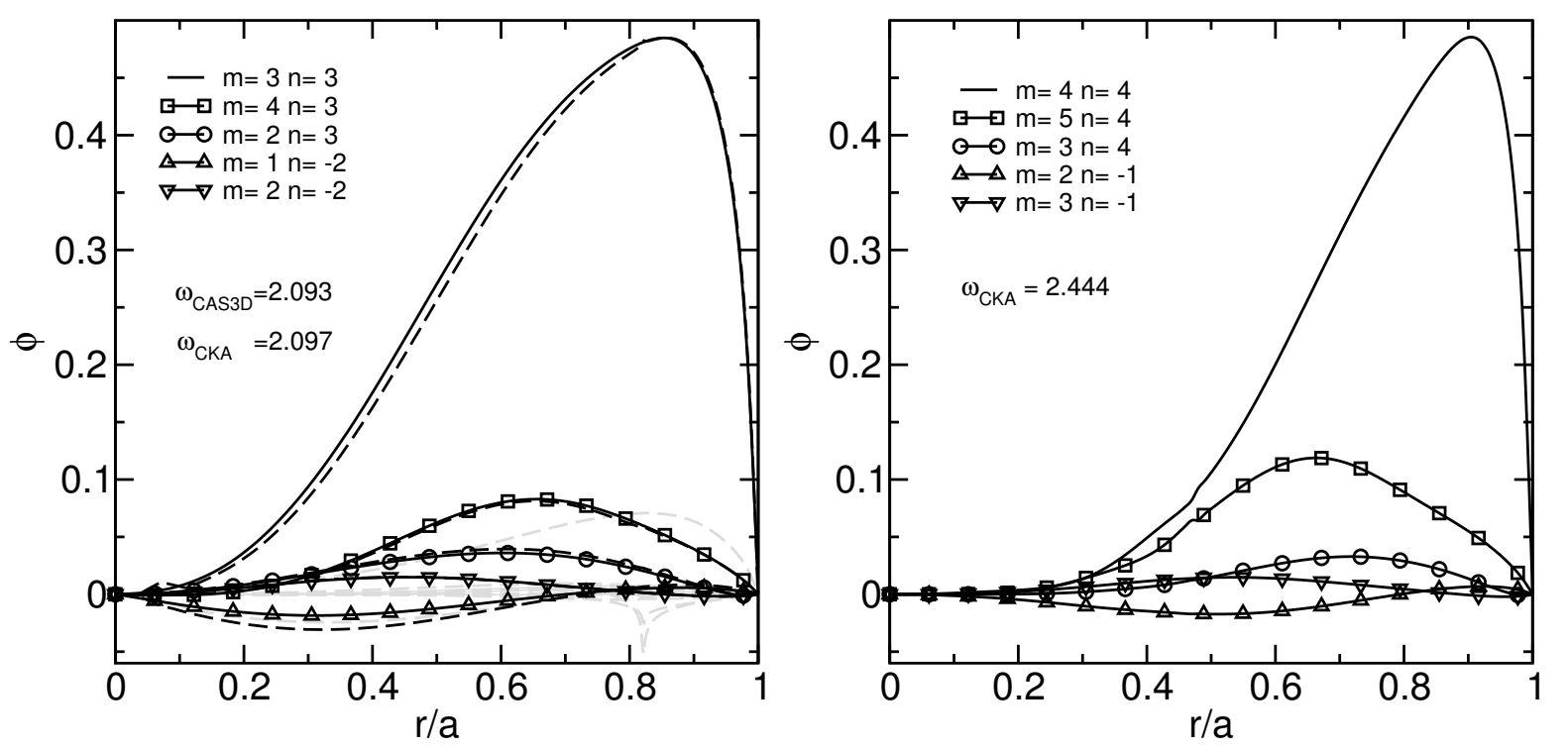

Figure 7. The modes with the dominant mode numbers $|m|=|n|=3$ (left panel) and $|m|=|n|=4$ (right panel) found by the CAS3D code (broken lines) without taking into account the plasma compressibility, and by the CKA code (solid lines), in the W7-X high mirror configuration in the absence of the energetic ions. The result is similar to the modes shown in figure 6 , which was calculated for the standard configuration in a compressible plasma without fast ions by solving equation (54).

compressibility and the presence of the energetic ions. This equation was derived and analyzed by perturbatively (analytically in the local approach) and non-perturbatively (numerically). In the absence of the energetic ions, similar results were obtained by the CAS3D and CKA codes.

- Both passing and trapped NBI ions can interact resonantly with IMs in W7-X. However, only the contribution of the passing ions was included in our analysis, but these constitute a majority of the fast-ion population in the plasma core. In addition, it seems that a considerable fraction of trapped energetic ions will be lost. The point is that $\beta$ in the first NBI experiments on W7-X will be not sufficient for good confinement of trapped fast ions [19]. The neoclassical electric field, being negative, is a favourable factor for their confinement but is not sufficient to improve the situation drastically. On the other hand, trapped NBI ions, if sufficiently well confined, may play an important role: first, they dominate in the plasma periphery where the amplitudes of the modes with $m / n=3 / 3,4 / 4$ are the largest (see figures 6 and 7), and second, as shown in Appendix A, they can interact with IMs having higher mode numbers (up to $m=n=6$ ) and, hence, higher mode frequencies, so that their ion Landau damping is less than that of the modes with $m=n \gtrsim 1$. Therefore, the study of the influence of trapped energetic ions on the IM modes and other modes is to be studied in future.

- To know IM damping is necessary for a reliable prediction of the NBI-driven IM instabilities in W7-X. One can expect that the IMs will damp through Landau mechanism mainly in the plasma core, where the phase velocity is smallest. Accordingly, their destabilization may have a favourable effect on the energy deposition of the energetic ions, channeling the energy of these ions (both passing and trapped ones) from the 
periphery region to plasma center. We recall that spatial channeling of the energy and momentum of energetic ions appears to have been observed in the NSTX spherical torus [16], where it was caused by high-frequency instabilities and had negative effect on the plasma balance. Moreover, these experiments [17] demonstrated that the efficiency of the energy transfer across the magnetic field by the destabilized modes well exceeded that due to plasma thermal conductivity. Calculating the damping, including its radial dependence, is therefore an important task for the future.

- Immediately after the NBI source is switched on, strong transient isomon instabilities, lasting for $\Delta t \ll \tau_{\mathcal{E}}$ and involving resonant particles with different pitch angles, may arise because there is a region with $\partial F / \partial \mathcal{E}>0$ in this case.

- It may be possible to excite global IMs by external antennas, channeling the RF

energy to the plasma core. This would confirm the predicted existence of the modes extending over a large part of the plasma cross section. In the case of positive result, it could serve as a method of the plasma heating with a low frequency $\left(\omega \ll \omega_{B i}\right)$ RF field, without employing the Alfvén resonance.

\section{Acknowledgments}

This work was supported in part by the Project No. 6058 of the Science and Technology Center in Ukraine and the National Academy of Sciences of Ukraine (NASU) and the Project No. 0114 U000678 of NASU.

The work has received funding from the European Unions Horizon 2020 research and innovation program under grant agreement number 633053. The views and opinions expressed herein do not necessarily reflect those of the European Commission. The work has been supported by EUROfusion Enabling Research grant CFPWP14ER011ENEAFrascati01.

\section{Appendix A. Energetic ions at the initial stage of operation with NBI in W7-X}

An injection power up to $6.84 \mathrm{MW}$ is planed for use in the first experiments with NBI on W7-X [18]. Balanced injection will be used, which will be provided by two pairs of injectors. The maximum energy of the injected atoms will be $55 \mathrm{keV}$ for hydrogen and $60 \mathrm{keV}$ for deuterium. Most experiments are planned with hydrogen. Pitch-angle characteristics of the injected particles are shown in figures A1 and A2.

We observe that both trapped and passing particles will be produced as a result of NBI. Passing particles dominate in the core region, whereas most trapped ones are born in the periphery of the plasma. Losses to the first wall are substantial for the trapped particles [19].

To investigate possible resonances between energetic ions and isomon modes, we first note that the resonance condition for the locally trapped particles is given by

$$
\omega=\omega_{b}, \quad \omega_{b}=N \frac{v}{R} \sqrt{\frac{\epsilon_{m}}{2}} .
$$



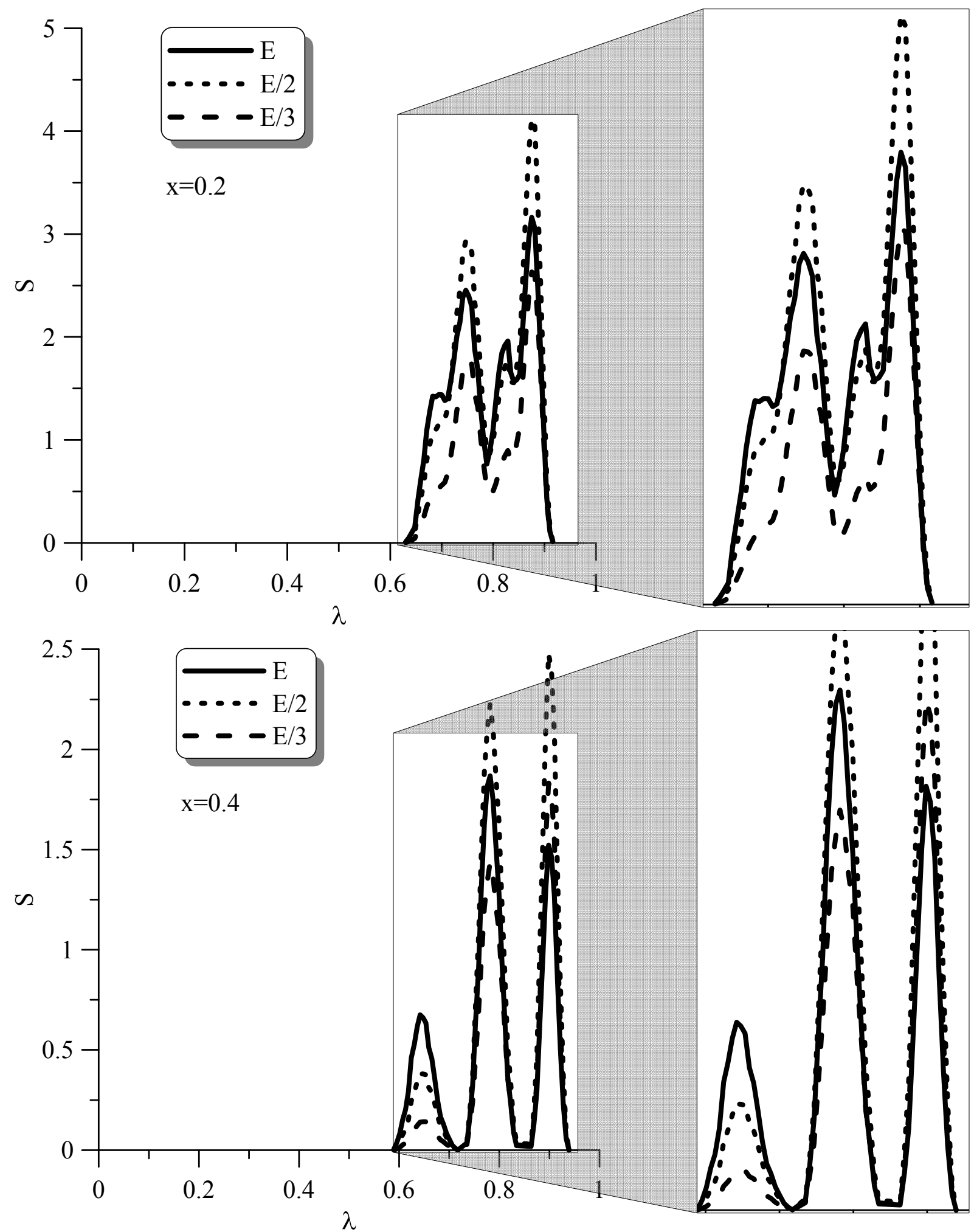

Figure A1. The $\lambda$ distribution of protons born by NBI at $x \equiv r / a=0.2$ and $x=0.4$. Here $S$ is a source function, $\lambda=\mu_{p} \bar{B} / \mathcal{E}$, and $\mu_{p}$ is the particle magnetic moment. 


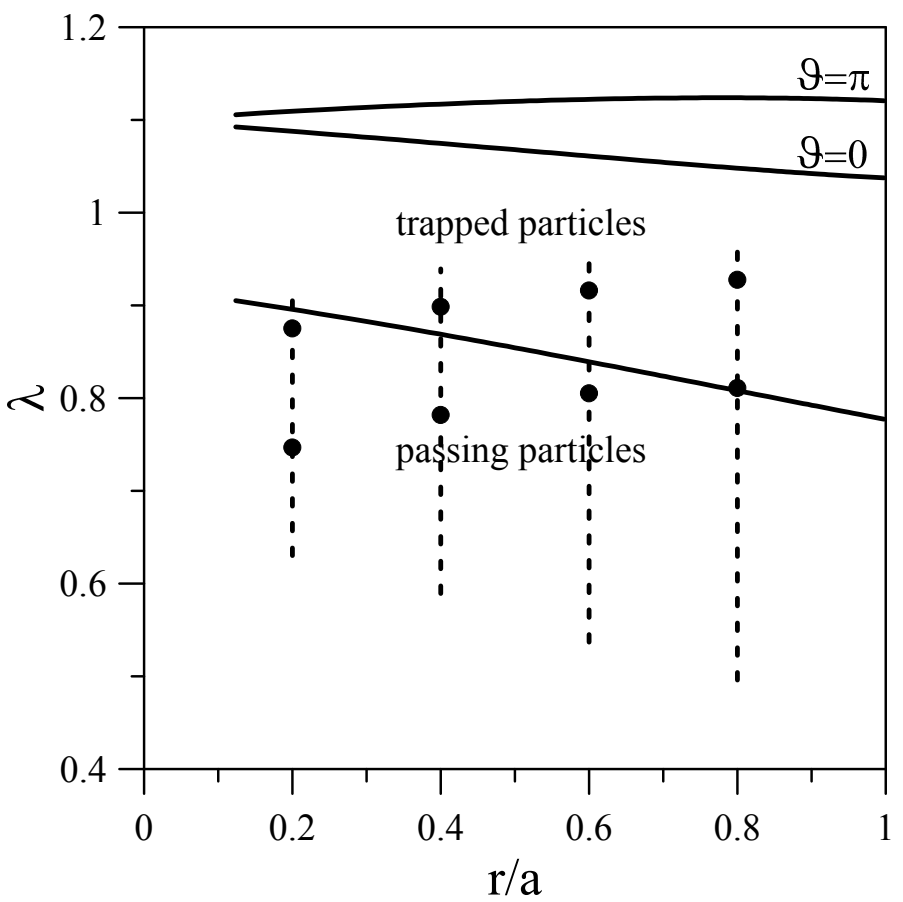

Figure A2. Regions of trapped particles (at $\vartheta=0$ and $\vartheta=\pi$ ) and passing particles in the $(\lambda, r / a)$ plane. Vertical dotted lines at several radii show the range of the NBI produced ions, the $\lambda$-location of maxima of the born ions on each line is shown by solid circles. The lowermost solid line represents the trapped-passing boundary, which is independent of theta.

For the high mirror configuration and $\omega \approx k_{m} v_{A}$, with $k_{m}=-m \Delta \iota / R$, this gives the resonant velocity

$$
v^{r e s} \approx v_{A} \frac{m \Delta \iota}{N} \sqrt{\frac{2}{\epsilon_{m}}}
$$

which for the used W7-X parameters $\left(N=5, v_{A} / v_{\alpha}=1.7, \epsilon_{m}=0.1\right.$, and $\left.\Delta \iota=0.1\right)$ simplifies to

$$
m=6.6 \frac{v^{r e s}}{v_{\alpha}}<7 .
$$

Passing particles interact with isomon modes through the resonance

$$
\omega=\left(k_{m} \pm \iota / R\right) v_{\|},
$$

which leads to the resonance pitch

$$
\chi^{r e s}=\frac{|m| \Delta \iota}{ \pm \iota-m \Delta \iota} \frac{v_{A}}{v} .
$$

Because $\chi<1$ and $v_{A}>v$, we conclude that (A.5) is satisfied when $m \ll \iota / \Delta \iota \sim 10$.

\section{Appendix B. The influence of the ambipolar electric field on the confinement of injected ions}

Numerical investigation of the confinement of injected protons predicts considerable collisionless losses of trapped particles for the time $\Delta t \lesssim 1 \mathrm{~ms}$ [19]. This time well exceeds 


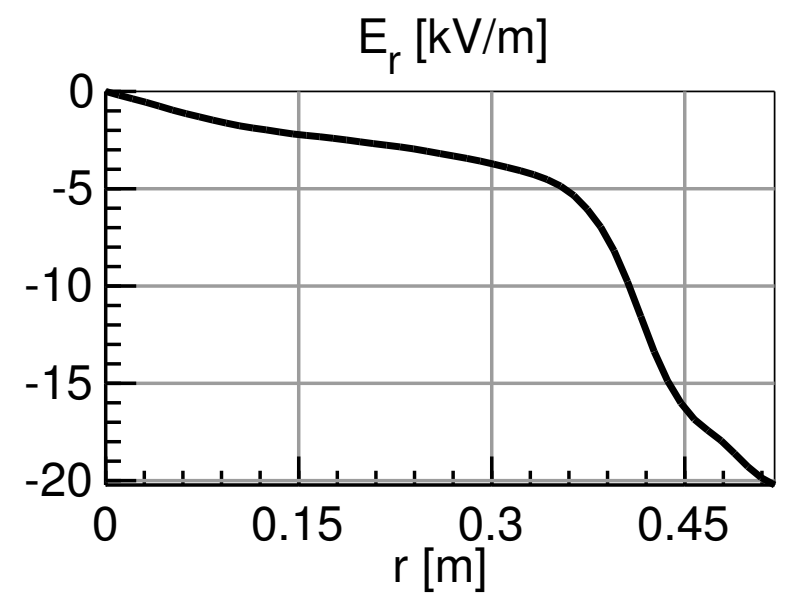

Figure B1. The radial electric field determined by neoclassical theory.
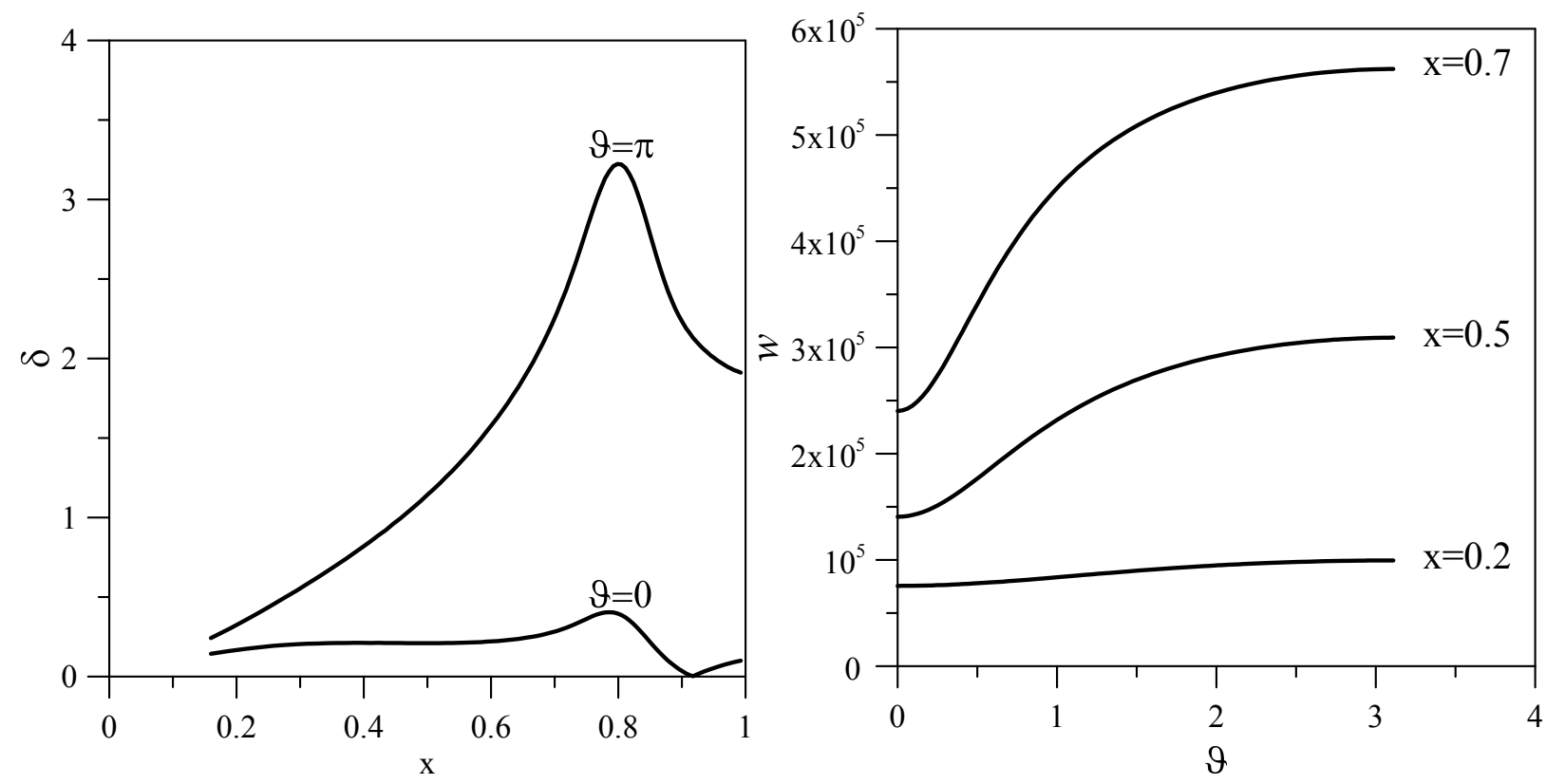

Figure B2. $\delta$ versus $x=r / a$ (left panel) and $w$ versus $\vartheta$ (right panel) for $\kappa \ll 1$ in the presence of a radial field (Figure B1).

the bounce period of locally trapped particles $\left(\tau^{L} \sim 2 \pi R /\left(N v \chi^{L}\right)=2.1 \times 10^{-6} / \chi^{L}\right.$ s, with $\chi^{L}$ a characteristic pitch of the particles), which is comparable to the particle precession time $\left(\tau_{p r} \sim 2 \pi r / v_{p r}\right.$, with $\left.v_{p r}=0.5 \rho v \partial \ln B / \partial r\right), \rho=v / \omega_{B \alpha}$. This indicates that particles escape from the plasma due to their regular motion along superbanana obits.

In addition, some losses were observed for $\Delta t>1 \mathrm{~ms}$ [19]. It seems that they can be explained by collisionless diffusion caused by separatrix-crossing of transitioning particles (trapped particles that undergo orbit transformation from the locally passing state to locally trapped state and vice versa) [20].

These results were obtained in the assumption that the radial electric field is absent. Therefore, the question arises how the radial electric field, which is usually present in the plasma, will affect the confinement of the energetic ions. Below we discuss this question, restricting ourselves to a consideration of locally trapped particles, whose confinement is 
the greatest concern [19].

According to reference [21], the motion of the locally trapped ions can be described by the following equations:

$$
\begin{aligned}
& \langle\dot{r}\rangle=u \sin \vartheta, \\
& r\langle\dot{\vartheta}\rangle=u \cos \vartheta+w,
\end{aligned}
$$

where

$$
\begin{aligned}
& u=\frac{v_{d}}{\epsilon}\left[\frac{\epsilon_{m} \epsilon_{h}}{\epsilon_{H}}\left(\frac{2 E(\kappa)}{K(\kappa)}-1\right)-\epsilon_{t}\right] \\
& w=\frac{v_{d}}{\epsilon}\left[r \epsilon_{0}^{\prime}-\frac{\epsilon_{h}^{2}}{\epsilon_{H}}\left(\frac{2 E(\kappa)}{K(\kappa)}-1\right)\right]+v_{E},
\end{aligned}
$$

$\langle(\ldots)\rangle$ means bounce averaging, $\mathbf{K}(\kappa)$ and $\mathbf{E}(\kappa)$ are the complete elliptic integrals of the first kind and second kind, respectively, $\kappa=\kappa(\vartheta)$ is the particle trapping parameter, $v_{E}$ is the poloidal component of the $\mathbf{E} \times \mathbf{B}$ drift velocity, $\epsilon_{h}=-\epsilon_{11}>0$ is the largest helical component of the equilibrium magnetic field, $\epsilon_{m}=\epsilon_{01}$ is the mirror component, $\epsilon_{H}(\vartheta)=\sqrt{\epsilon_{m}^{2}+\epsilon_{h}^{2}-2 \epsilon_{m} \epsilon_{h} \cos \vartheta}$, and $\epsilon_{0}=0.5 \epsilon_{00}$ describes the effect of diamagnetism, $v_{d}=\rho v /(2 R)$.

It follows from (B.1), (B.2) that good confinement of the particles could be achieved if

$$
\delta \equiv|w / u| \gg 1 \text { and } w(\vartheta) \approx \text { const. }
$$

In the absence of the electric field these conditions can be satisfied only due to the term proportional to $\epsilon_{0}^{\prime}$, which requires sufficiently high $\beta$. Note that the necessity of high $\beta$ for good confinement of energetic ions was shown for the first time numerically in [22] and remains a key point in the optimization of Wendelstein-line stellarators. However, at the initial stage of operation of W7-X the injected power will be not sufficient to provide the required $\beta$. One can see that the diamagnetic term in (B.4) is smaller than the helical term in a wide range of poloidal angles. This implies that most of the locally trapped particles will be lost.

In most plasma scenarios, the calculated radial electric field determined by the ambipolarity condition of the neoclassical diffusion of electrons and ions is negative (figure B1). This field, as seen from equation (B.4), tends to improve particle confinement due to the fact that $v_{E}$ has the same sign as $\epsilon_{0}^{\prime}$. However, there is a question whether this field is strong enough to satisfy the condition given by (B.5). Figure B2 gives the answer. We observe that $\delta>1$ in the periphery region $(r / a>0.5)$ of the inner circumference of the torus but is less then unity at small poloidal angles. In addition, we observe that $w(\vartheta)$ is approximately constant only for $r / a \ll 1$. Therefore, we conclude that the positive effect of the neoclassical electric field is rather small in the considered case.

\section{References}

[1] Weller A., Anton M., Geiger J. et al 2001 Phys. Plasmas 8931

[2] Weller A., Geiger J., Werner A. et al 2003 Plasma Phys. Control. Fusion 45 A285 
[3] Hirsch M., Baldzuhn J., Beidler C., et al 2008 Plasma Phys. Control. Fusion 50053001

[4] Beidler C. D., Harmeyer E., Herrnegger F., et al 2001 Nucl. Fusion 411759

[5] Kolesnichenko Ya. I., Lutsenko V.V., Weller A., Werner A., Yakovenko Yu. V., Geiger J., Fesenyuk O. P. 2007 Phys. Plasmas 14102504

[6] Kolesnichenko Ya. I., Könies A., Lutsenko V. V. and Yakovenko Yu. V. 2011 Plasma Phys. Control. Fusion 53024007

[7] Gorelenkov N. N., Berk H. L., Crocker N. A. et al 2007 Plasma Phys. Control. Fusion 49 B371

[8] Fesenyuk O. P., Kolesnichenko Ya.I., Yakovenko Yu.V. 2012 Plasma Phys. Control. Fusion 54 085014

[9] Kolesnichenko Ya. I., Lepiavko B. S., Lutsenko V. V. 2013 Plasma Phys. Control. Fusion 55125007

[10] Kolesnichenko Ya. I., Lutsenko V. V., Wobig H., Yakovenko Yu. V., Fesenyuk O. P. 2001 Phys. Plasmas 8491

[11] Kolesnichenko Ya. I., Lutsenko V. V., Wobig H., Yakovenko Yu. V. 2002 Phys. Plasmas 9517

[12] Kolesnichenko Ya. I., Yamamoto S., Yamazaki K., Lutsenko V. V., Nakajima N., Narushima Y., Toi K, Yakovenko Yu V 2004 Phys. Plasmas 11158

[13] Kolesnichenko Ya. I. 1980 Nucl. Fusion 20727

[14] Schwab C. 1993 Phys. Fluids B 53195

[15] Könies A. 2007 "A code for the calculation of kinetic Alfvén waves in three-dimensional geometry", 10th IAEA TM on Energetic Particles in Magnetic Confinement Systems, http://www.ipp.mpg.de/iaea_2007, P15

[16] Kolesnichenko Ya. I., Yakovenko Yu. V., Lutsenko V. V. 2010 Phys. Rev. Lett. 104075001

[17] Stutman D. et al. 2009 Phys. Rev. Lett. 102115002

[18] Klinger T., Baylard C., Beidler C. D., Boscary J., Bosch H. S., Dinklage A., Hartmann D., Helander P., Maßberg H., Peacock A., Pedersen T. S., Rummel T., Schauer F., Wegener L., and Wolf R. 2013 Fusion Engineering and Design 88461

[19] Drevlak M., Geiger J., Helander P., Turkin Y. 2014 Nucl. Fusion 54073002

[20] Beidler C. D. , Kolesnichenko Ya. I., Marchenko V. S., Sidorenko I. N., Wobig H. 2001 Phys. Plasmas 82731

[21] Kolesnichenko Ya. I., Lutsenko V. V., Tykhyy A. V., Weller A., Werner A., Wobig H., Geiger J. 2006 Phys. Plasmas 13072504

[22] Lotz W., Merkel P., Nührenberg J., and Strumberger E. 1992 Plasma Phys. Control. Fusion 34 1037 